\title{
Neurally-Adjusted Ventilatory Assist for Noninvasive Ventilation via a Helmet in Subjects With COPD Exacerbation: A Physiologic Study
}

\author{
Federico Longhini, Ling Liu, Chun Pan, Jianfeng Xie, Gianmaria Cammarota, Andrea Bruni, \\ Eugenio Garofalo, Yi Yang, Paolo Navalesi, and Haibo Qiu
}

\begin{abstract}
BACKGROUND: In patients with COPD exacerbation, noninvasive ventilation (NIV) is strongly recommended. NIV is generally delivered by using patient triggered and flow-cycled pressure support through a face mask. A specific method to generate neurally-controlled pressure support has been shown to improve comfort and patient-ventilator interaction. In addition, the helmet interface was better tolerated by patients compared with a face mask. Herein, we compared neurally-controlled pressure support through a helmet with pressure support through a face mask with respect to subject comfort, breathing pattern, gas exchange, pressurization and triggering performance, and patientventilator synchrony. METHODS: Two 30-min trials of NIV were randomly delivered to 10 subjects with COPD exacerbation redundant: (1) pressure support through a face mask with inspiratory pressure support of $\geq 8 \mathrm{~cm} \mathrm{H} \mathrm{H}_{2} \mathrm{O}$ to obtain a tidal volume of 6-8 $\mathrm{mL} / \mathrm{kg}$ of ideal body weight; and (2) NAVA through a helmet, setting the neurally-adjusted ventilatory assist level at $15 \mathrm{~cm} \mathrm{H}_{2} \mathrm{O} / \mu \mathrm{V}$, with an upper airway pressure limit to obtain the same overall airway pressure applied during pressure support through a face mask. We assessed subject comfort, breathing frequency, respiratory drive, arterial blood gases, pressure-time product (PTP) of the first $300 \mathrm{~ms}$ and $500 \mathrm{~ms}$ after initiation of subject effort, inspiratory trigger delay, and rate of asynchrony determined as the asynchrony index. RESULTS: Median and interquartile range NAVA through a helmet improved comfort (7.0 [6.0-8.0]) compared with pressure support through a face mask $(5.0[4.7-5.2], P=.005)$. The breathing pattern was not different between the methods. Respiratory drive was slightly, although not significantly, reduced $(P=.19)$ during NAVA through a helmet in comparison with pressure support through a face mask. Gas exchange was also not different between the trials. The PTP of the first $300 \mathrm{~ms}(P=.92)$ and PTP of the first $500 \mathrm{~ms}(P=.08)$ were not statistically different between trials, whereas triggering performance, patient-ventilator interaction, and synchrony were all improved by NAVA through a helmet compared with pressure support through a face mask. CONCLUSIONS: In the subjects with COPD with exacerbation, NAVA through a helmet improved comfort, triggering performance, and patient-ventilator synchrony compared with pressure support through a face mask. Key words: noninvasive ventilation; mechanical ventilation; pressure-support ventilation; neurally adjusted ventilatory assist; patient-ventilator interaction; ventilator performance; patient-ventilator asynchrony. [Respir Care 2019;64(5):582-589. () 2019 Daedalus Enterprises]
\end{abstract}

\section{Introduction}

In patients with COPD exacerbation and respiratory acidosis, noninvasive ventilation (NIV) is strongly recommended as a first-line treatment. ${ }^{1}$ Most commonly, NIV is applied through a face mask by using a pneumatically triggered and flow-cycled pressure support modality. ${ }^{2} \mathrm{How}-$ ever, a face mask can be an uncomfortable interface, and pressure support does not guarantee good patient-ventilator interaction and potentially leads to NIV failure. ${ }^{3,4}$

The helmet is a well-tolerated interface, which allows prolonged application without NIV discontinuation ${ }^{5}$ and improved outcomes in patients with ARDS. ${ }^{6}$ However, the helmet is characterized by poorer patient-ventilator interaction and pressurization performance compared with a face mask. ${ }^{7}$ Such drawbacks have been partially improved in a new-generation helmet that introduces some technical advances. ${ }^{8} 9$ In particular, the new-generation helmet is characterized by an annular openable ring placed underneath an inflatable cushion that secures the helmet without the need for armpit braces. Such technical advances have improved patient-ventilator interactions by ameliorating the triggering phase and pressurization performance. ${ }^{89}$ In subjects with COPD exacerbation, the new-generation hel- 
met has also been found to be an effective interface to improve alveolar ventilation, while also achieving comfort similar to a face mask. ${ }^{10}$

Recently, a specific method of applying neurally-adjusted ventilatory assist has been proposed to generate neurally-controlled pressure support in patients who receive either invasive ventilation ${ }^{11}$ or NIV through a helmet ${ }^{12}$ or a face mask. ${ }^{13}$ Compared with pressure support, neurally-controlled pressure support results in better pressurization and triggering performance, and improves patient comfort without affecting gas exchange during NIV. ${ }^{12,13}$ Moreover, NAVA improves patient-ventilator interaction and triggering performance in patients with COPD exacerbation who are intubated when compared with pressure support. ${ }^{11}$

We hypothesized that the application of neurally-controlled pressure support through a new generation helmet could improve patient comfort compared with cycled-off pressure support through a face mask. This physiologic study aimed to compare NAVA through a helmet with cycled-off pressure support through a face mask with respect to comfort (primary end point), breathing pattern, respiratory drive, gas exchange, pressurization and triggering performance, and patient-ventilator synchrony (additional end points) in subjects with a COPD exacerbation who were receiving NIV.

\section{Methods}

The present physiologic, crossover, randomized study was conducted from August to December 2013 in the ICU, Department of Critical Care Medicine, ZhongDa Hospital, Southeast University, School of Medicine, Nanjing, China. The study was approved by the research ethics board of Zhongda Hospital, Southeast University, Nanjing, China (2013ZDSYLL097.0). Written informed consent was obtained from the subjects for publication of their individual details and accompanying images in this article. At the time that the study was conducted, trial registration was not mandatory for this type of investigation.

\section{Subjects}

We considered eligible any adult patient with a previous diagnosis of COPD (ie, the presence of dyspnea, chronic cough or sputum production, and/or a history of exposure to risk factors, with a post-bronchodilator $\mathrm{FEV}_{1} / \mathrm{FVC}$ of

\footnotetext{
Drs Longhini, Bruni, Garofalo, and Navalesi are affiliated with the Intensive Care Unit, University Hospital Mater Domini, Department of Medical and Surgical Sciences, Magna Graecia University, Catanzaro, Italy. Drs Liu, Pan, Xie, Yang, and Qiu are affiliated with the Department of Critical Care Medicine, Zhongda Hospital, Southeast University, School of Medicine, Nanjing, China. Dr Cammarota is affiliated with the
}

\section{QUICK LOOK}

\section{Current knowledge}

Patients with COPD exacerbation and with respiratory acidosis require NIV as first-line treatment. To reduce NIV failure, clinicians attempt to ensure optimal comfort by the choice of a proper interface and ventilator settings. The helmet has been shown to improve patient tolerance to the interface compared with a mask. NAVA has been shown to further improve comfort and patientventilator interaction, and to reduce respiratory drive.

\section{What this paper contributes to our knowledge}

NAVA through a helmet was more comfortable compared with conventional pneumatically-triggered pressure support through a mask in a group of subjects with COPD exacerbation. Furthermore, as opposed to pressure support through a face mask, NAVA through a helmet improved patient-ventilator interaction and synchrony, without affecting the respiratory drive and pattern.

$<0.70$ at the spirometry ${ }^{14}$ ), admitted to the ICU for exacerbation and acute respiratory failure, which we defined as $\mathrm{pH}<7.35$, with $\mathrm{P}_{\mathrm{aCO}}>45 \mathrm{~mm} \mathrm{Hg}$ while breathing room air or with oxygen supplementation via a air-entrainment mask and required NIV. ${ }^{10,14}$

The inclusion criteria were the following: (1) the subject was fully cooperative; (2) no infusion of midazolam and propofol in the previous 24 and $4 \mathrm{~h}$, respectively; (3) PEEP $\geq 8 \mathrm{~cm} \mathrm{H}_{2} \mathrm{O}$, with a total applied pressure (ie, PEEP plus inspiratory support) of $\leq 25 \mathrm{~cm} \mathrm{H}_{2} \mathrm{O}$; (4) arterial $\mathrm{pH}>7.34$ during NIV; and (5) breathing frequency $\leq 30$ breaths $/ \mathrm{min}$. The exclusion criteria were as

Anesthesia and Intensive Care, "Maggiore della Carità" Hospital, Novara, Italy.

Drs Longhini and Liu are co-first authors.

The study was conducted in the Intensive Care Unit, Department of Critical Care Medicine, Zhongda Hospital, Southeast University, School of Medicine, Nanjing, China.

Dr Navalesi discloses relationships with Intersurgical S.p.A., Maquet Critical Care, Hillrom, Philips, Resmed, and Novartis. The remaining authors have disclosed no conflicts of interest.

Correspondence: Federico Longhini MD, Intensive Care Unit, University Hospital Mater Domini, Department of Medical and Surgical Sciences, Magna Graecia University Viale Europa, 88100, Catanzaro, Italy. E-mail: longhini.federico@gmail.com.

DOI: $10.4187 /$ respcare. 06502 
follows: (1) the need for analgesic or sedative drugs, (2) recent cervical spine injury, (3) obstructive sleep apnea syndrome, (4) claustrophobia, (5) contraindications to placement of a nasal-gastric feeding tube, (6) face or neck deformities, (7) pregnancy, (8) inclusion in other research protocols, (9) and lack of consent. ${ }^{7,13}$

\section{Study Protocol}

After each subject's enrollment in the study, NAVA catheter (Maquet Critical Care, Solna, Sweden) was placed in the esophagus and correct positioning was ascertained as previously described. ${ }^{15}$ The study was performed by using a standard Servo-i ventilator (Maquet Critical Care) equipped with NAVA module and NIV software for air leaks. All the subjects subsequently underwent two 30-min trials (pressure support through a face mask and NAVA support through a helmet) in random order according to a computer-generated random sequence by using sealed, opaque, numbered envelopes. The envelopes were kept in the head nurse's office. The envelope was opened by the nurse in charge of the subject, and the prescribed sequence of modes was communicated to the investigators.

Settings in both modes of ventilation were titrated to obtain the same ventilatory support provided to the subjects before study entry. In particular, the pressure support through a face mask trial was conducted through a face mask individually selected for each subject based on his or her anthropometric characteristics to minimize air leaks and optimize subject tolerance ${ }^{13}$; the face mask was selected from among 2 different models: FreeMotion RT041 Non Vented Full Face Mask (Fisher and Paykel, Auckland, New Zealand) and PerforMax Face Mask (Philips Respironics, Murrysville, Pennsylvania). The ventilator was set as previously clinically indicated by the attending physician. In particular, inspiratory pressure support was $\geq 8 \mathrm{~cm} \mathrm{H}_{2} \mathrm{O}$ to obtain a tidal volume of $6-8 \mathrm{~mL} / \mathrm{kg}$ of ideal body weight, with the fastest rate of pressurization and cycling that was between 25 and $50 \%$ of peak inspiratory flow. ${ }^{10}$

The NAVA through a helmet trial was conducted with a new-generation helmet (Castar Next, Intersurgical, Mirandola, Italy) in NAVA, with the NAVA level set at its maximum (ie, $15 \mathrm{~cm} \mathrm{H}_{2} \mathrm{O} / \mu \mathrm{V}$ ), the same PEEP applied during the pressure support through a face mask trial and an upper airway pressure $\left(\mathrm{P}_{\mathrm{aw}}\right)$ limit to obtain the same overall $\mathrm{P}_{\mathrm{aw}}$ applied during the pressure support through a face mask trial. ${ }^{11,16,17}$ The trigger sensitivity was set at $0.5 \mu \mathrm{V}$, whereas the default cycling was $70 \%$ of the peak electrical activity of the diaphragm $\left(\mathrm{EA}_{\mathrm{di}}\right)$, as fixed by the company. ${ }^{16} \mathrm{~F}_{\mathrm{IO}_{2}}$ was set to maintain peripheral $\left(\mathrm{S}_{\mathrm{pO}_{2}}\right)$ between $90 \%$ and $94 \%,{ }^{14}$ and remained unmodified throughout the study period.
Predefined criteria for protocol interruption were the following: (1) the need for emergency intubation to protect the airway; (2) $\mathrm{S}_{\mathrm{pO}_{2}}$ of $<86 \%$; (3) acute respiratory acidosis, as defined by $\mathrm{P}_{\mathrm{aCO}_{2}}>50 \mathrm{~mm} \mathrm{Hg}$ and $\mathrm{pH}<7.30$; (4) an inability to expectorate secretions; (5) hemodynamic instability (ie, a need for continuous infusion of dopamine or dobutamine of $>5 \mu \mathrm{g} / \mathrm{kg} / \mathrm{min}$, norepinephrine of $>0.1 \mu \mathrm{g} / \mathrm{kg} / \mathrm{min}$, or vasopressin to maintain mean arterial blood pressure of $>60 \mathrm{~mm} \mathrm{Hg}$ ); (6) life-threatening arrhythmias or electrocardiographic signs of ischemia; or (7) Glasgow coma scale decline of $\geq 2$ points.

\section{Data Acquisition and Analysis}

Air flow, $\mathrm{P}_{\mathrm{aw}}$, and $\mathrm{Ea}_{\mathrm{di}}$ were acquired from the ventilator through an RS232 interface at a sampling rate of $100 \mathrm{~Hz}$ and were recorded on a computer with dedicated software (ServoTracker V. 4.0, Maquet Critical Care). The last minute of each trial was manually analyzed off-line by using customized software based on Microsoft Excel Microsoft, Redmond, WA, as previously described. ${ }^{18}$ Comfort was assessed through an 11-point numeric rating scale, as previously reported.9,13,17 Before protocol initiation, all the subjects received a detailed explanation of the numeric rating scale. The subjects evaluated their comfort level at the end of each trial with a number between 0 (worst possible comfort) and 10 (best possible comfort) by using an ICU-adapted large printed scale that included numbers and descriptors. The scores obtained were recorded without additional indications or comments. ${ }^{9,13,17}$ At the end of each trial, arterial blood was also sampled for gas analysis.

Mechanical inspiratory time $\left(\mathrm{T}_{\mathrm{I}}\right)$ and ventilator rate were determined from the flow tracing. Subject's own (neural) breathing frequency and neural $\mathrm{T}_{\mathrm{I}}$ were computed from the $\mathrm{EA}_{\mathrm{di}}$ tracing. Subjects' own (neural) breathing frequency and $\mathrm{T}_{\mathrm{I}}$ were computed from the $\mathrm{EA}_{\mathrm{di}}$ tracing. Mechanical $\mathrm{T}_{\mathrm{I}} / \mathrm{T}_{\text {tot }}$ and neural $\mathrm{T}_{\mathrm{I}} / \mathrm{T}_{\text {tot }}$ inspiratory duty cycle were calculated as the ratio between mechanical $\mathrm{T}_{\mathrm{I}} / \mathrm{T}_{\text {tot }}$ and the ratio between neural $\mathrm{T}_{\mathrm{I}} / \mathrm{T}_{\text {tot }}$, respectively. ${ }^{12,13,19}$ Leaks were computed as the difference between the volume insufflated to the interface and the exhaled volume back to the ventilator multiplied by the (ventilator rate; leaks were expressed as the rate of the inhaled volume over 1 min..$^{13,19}$ Moreover, we measured the peak $\mathrm{P}_{\mathrm{aw}}$, the peak inspiratory flow, and the time to reach peak inspiratory flow from the onset of the subject effort. ${ }^{13}$ The peak $\mathrm{EA}_{\mathrm{di}}$ was also calculated as the swing from baseline to its peak to assess the respiratory drive. ${ }^{12,13}$

The pressurization performance was assessed with the $\mathrm{P}_{\mathrm{aw}}$-time product (PTP) of the first $200 \mathrm{~ms}$ computed from the onset of ventilator assistance $\left(\mathrm{PTP}_{200}\right)$, excluding the triggering phase, and with the PTP of the first 300 and 500 $\mathrm{ms}$ from the onset of subject effort, indexed to the ideal 
Table 1. Subject Characteristics

\begin{tabular}{|c|c|c|c|c|c|c|c|c|c|}
\hline $\begin{array}{l}\text { Subject } \\
\text { No. }\end{array}$ & Sex & Age (y) & Weight (kg) & BMI $\left(\mathrm{kg} / \mathrm{m}^{2}\right)$ & $\begin{array}{l}\text { APACHE II } \\
\text { Score }\end{array}$ & $\begin{array}{c}\text { PEEP } \\
\left(\mathrm{cm} \mathrm{H}_{2} \mathrm{O}\right)\end{array}$ & $\begin{array}{c}\mathrm{PS} \\
\left(\mathrm{cm} \mathrm{H}_{2} \mathrm{O}\right)\end{array}$ & $\mathrm{F}_{\mathrm{IO}_{2}}$ & $\mathrm{FEV}_{1}(\%)$ \\
\hline 1 & M & 75 & 65 & 23.0 & 21 & 10 & 10 & 0.30 & 48 \\
\hline 2 & M & 73 & 60 & 23.4 & 28 & 12 & 12 & 0.40 & 47 \\
\hline 3 & M & 78 & 70 & 24.8 & 23 & 10 & 12 & 0.30 & 37 \\
\hline 4 & M & 68 & 56 & 20.6 & 25 & 10 & 12 & 0.45 & 40 \\
\hline 5 & M & 74 & 60 & 23.1 & 25 & 10 & 12 & 0.30 & 42 \\
\hline 6 & M & 83 & 72 & 24.9 & 22 & 11 & 13 & 0.25 & 35 \\
\hline 7 & $\mathrm{~F}$ & 86 & 60 & 27.3 & 22 & 8 & 14 & 0.30 & 38 \\
\hline 8 & M & 77 & 70 & 22.9 & 19 & 9 & 12 & 0.23 & 46 \\
\hline 9 & M & 70 & 70 & 24.2 & 10 & 9 & 12 & 0.23 & 36 \\
\hline 10 & M & 68 & 80 & 26.1 & 15 & 9 & 10 & 0.40 & 33 \\
\hline \multicolumn{10}{|c|}{$\begin{array}{l}\text { BMI }=\text { body mass index } \\
\text { APACHE II = Acute Physiology and Chronic Health Evaluation II } \\
\text { PS = inspiratory pressure support above PEEP }\end{array}$} \\
\hline
\end{tabular}

PTP (index PTP $_{300}$ and index $\mathrm{PTP}_{500}$, respectively). . $^{8,12,13,20}$ The ideal PTP was computed by considering a perfectly squared rectangle on the $\mathrm{P}_{\text {aw }}$-time tracing, with the height of the actual $\mathrm{P}_{\mathrm{aw}}$ above PEEP and the width of the time window considered (ie, 0.3 and $0.5 \mathrm{~s}$ from the onset of the inspiratory effort, assessed from the $\mathrm{EA}_{\mathrm{di}}$ tracing, for index $\mathrm{PTP}_{300}$ and index $\mathrm{PTP}_{500}$, respectively). ${ }^{8,12,13,20}$ The inspiratory trigger delay and the expiratory trigger delay, the PTP during the triggering phase, and the drop in $\mathrm{P}_{\mathrm{aw}}$ were computed to evaluate the triggering performance. $8,12,13,20$

To assess patient-ventilator synchrony, we calculated the time of synchrony between diaphragm activity and ventilator assistance indexed to the subject's own neural $\mathrm{T}_{\mathrm{I}}{ }^{8,12,13,20}$ Asynchronies (ineffective efforts, auto triggering and double triggering) were also assessed and expressed as the asynchrony index, that is, the total number of asynchronous events divided by the number of triggered and not triggered breaths. ${ }^{4}$ The asynchrony index of $\geq 10 \%$ was considered to indicate a clinically relevant rate of asynchronies. ${ }^{4}$

\section{Statistical Analysis}

Based on preliminary data, to ascertain an average increase in comfort of 2.0 with an expected SD of 2.0 with $\alpha$ risk of 0.05 and $\beta$ risk of 0.20 , a sample of 10 subjects was deemed necessary. ${ }^{12}$ Data were reported as median and $25-75 \%$ interquartile, unless otherwise specified. All continuous variables were compared by the Wilcoxon signed-rank test. We compared categorical data by using the Fisher exact test, whereas the Spearman rank correlation test was used to determine the correlation between each individual comfort score and the corresponding $\mathrm{PTP}_{200}$, index $\mathrm{PTP}_{300}$, index $\mathrm{PTP}_{500}$, PTP during the trig- gering phase, inspiratory trigger delay, peak inspiratory flow, and the time to reach peak inspiratory flow. We considered significant 2 -sided $P<.05$. All the statistical analyses were performed by using the SigmaPlot v. 12.0 (Systat Software, San Jose, California).

\section{Results}

We enrolled 10 consecutive subjects with COPD exacerbation. All the subjects completed the study protocol without any complication and were included in the data analysis. The subjects' demographic and anthropometric characteristics are shown in Table 1.

\section{Comfort}

The individual values of the comfort score for all the subjects and their median and interquartile range are depicted in Figure 1. The median and interquartile range NAVA through a helmet slightly, although significantly, improved comfort (7.0 [6.0-8.0]) compared with pressure support through a face mask (5.0 [4.7-5.2]) $(P=.005)$. Comfort improvement was not correlated to $\mathrm{PTP}_{200}$ $(\rho=-0.286, P=.01)$, index $\operatorname{PTP}_{300}(\rho=0.286, P=.22)$, index $\mathrm{PTP}_{500}(\rho=0.061, P=.39)$, PTP during the triggering phase $(\rho=0.373, P=.12)$, inspiratory trigger delay $(\rho=-0.423, P=.14)$, peak inspiratory flow ( $\rho=0.292, P=.21)$, and time to reach peak inspiratory flow $(\rho=0.007, P=.67)$.

\section{Breathing Pattern, Respiratory Drive, and Gas Exchange}

As reported in Table 2, the breathing pattern was not different between the modes. Air leaks were higher during 


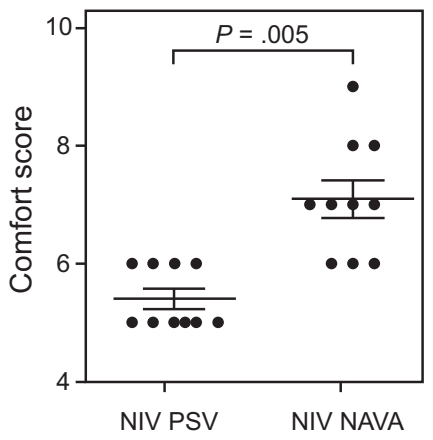

Fig. 1. Individual values (circles), median and interquartile range (solid lines) of the comfort score during pneumatically triggered pressure support through a mask (NIV PSV) and neurally controlled pressure support through a helmet (NIV NAVA).

pressure support through a face mask as opposed to NAVA through a helmet $(P<.01)$. Compared with pressure support through a face mask, NAVA through a helmet was characterized by a higher peak inspiratory flow $(P<.01)$, whereas the time to reach peak inspiratory flow was similar between the modes $(P=.16)$.

Shown in Figure 2, from top to bottom, $\mathrm{P}_{\mathrm{aw}}$, flow, and $\mathrm{EA}_{\mathrm{di}}$ tracings of one representative subject who underwent pressure support through a face mask (left) and NAVA through a helmet (right). The arrow indicates an ineffective inspiratory effort during pressure support through a face mask. The median group values are presented in Table 2 . Respiratory drive was slightly, although not significantly, reduced $(P=.19)$ during NAVA through a helmet in comparison with pressure support through a face mask. Gas exchange was also not different between the trials (Table 2).

\section{Pressurization and Triggering Performance}

Pressurization and triggering performance data are reported in Table 2. Compared with NAVA through a helmet, $\mathrm{PTP}_{200}$ was significantly higher during cycled-off pressure support through a face mask $(P<.01)$, whereas index $\operatorname{PTP}_{300}(P=.92)$ and index $\operatorname{PTP}_{500}(P=.08)$ were not statistically different between the trials. Shown also in Table 2, neurally controlled pressure support through a helmet significantly reduced inspiratory trigger delay $(P=.037)$, PTP during the triggering phase $(P<.01)$, and the drop in $\mathrm{P}_{\mathrm{aw}}(P=.049)$, in contrast to pressure support through a face mask. Inspiratory trigger delay was no different between the modes $(P=.16)$.

\section{Patient-Ventilator Synchrony}

Compared with pressure support through a face mask, NAVA through a helmet did not improve the patient-ventilator synchrony to neural $\mathrm{T}_{\mathrm{I}}$ ratio (Table 2). As expected, during NAVA through a helmet, the asynchrony index \% was $<10 \%$ in all subjects, whereas it was $\geq 10 \%$ in 6 subjects $(60 \%)$ with pressure support through a face mask $(P=.01)$. The median and interquartile range of the asynchrony index were $13.51 \%(2.6-52.9 \%)$ during pressure support through a face mask and $0 \%(0-0 \%)$ during NAVA through a helmet $(P<.01)$.

\section{Discussion}

This physiologic study shows that, in subjects with COPD who received NIV because of exacerbation and acute respiratory failure compared with pressure support through a face mask, NAVA through a helmet improved comfort, patient-ventilator synchrony, and triggering performance, whereas it did not affect respiratory drive, pressurization performance, and gas exchange.

To the best of our knowledge, this investigation was the first to evaluate NAVA for delivery of NIV through a helmet to subjects with COPD and with exacerbation. In a study that evaluated subjects with COPD and who were intubated and intrinsic PEEP, neurally controlled pressure support improved patient-ventilator interaction and synchrony, and counterbalanced the extra load due to intrinsic PEEP without the need for externally applied PEEP compared with pressure support. ${ }^{11}$ In 2 recent studies conducted in subjects considered to be at risk for extubation failure, NAVA improved patient-ventilator synchrony and pressurization and triggering performance, thereby improving the subject's comfort during NIV through a helmet ${ }^{12}$ and mask. ${ }^{13}$

Consistent with the results of these investigations, in the present study, NAVA through a helmet outperformed pressure support through a face mask with respect to comfort, triggering performance, and patient-ventilator synchrony. However, although index $\mathrm{PTP}_{300}$ and index $\mathrm{PTP}_{500}$ were similar between modes, $\mathrm{PTP}_{200}$ was higher during pressure support through a face mask compared with NAVA through a helmet. These data were in contrast with those reported in previous studies. ${ }^{12,13}$ This was likely due to the different physical properties of a mask and a helmet, the latter being characterized by more problematic pressurization performance. Furthermore, this may have also affected the slight, although not significant, reduction of the respiratory drive.

Nonetheless, compared with pressure support through a face mask, NAVA through a helmet improved comfort, which is a major determinant of NIV outcome. In contrast to previous studies, ${ }^{12,13}$ we did not find any correlation between comfort and pressurization or triggering performance. Such improvement may mainly be due to the applied interface (ie, the helmet); in fact, the helmet has been largely recognized to be comfortable $5,9,10$ and to reduce intubation rates ${ }^{6}$ and in-hospital 90-d and 
Table 2. Breathing Pattern, Respiratory Drive, Gas Exchange, Pressurization and Triggering Performance, and Patient-Ventilator Synchrony

\begin{tabular}{|c|c|c|c|}
\hline Parameter & $\begin{array}{c}\text { Pressure Support } \\
\text { Through a Face Mask }\end{array}$ & NAVA Through a Helmet & $P$ \\
\hline \multicolumn{4}{|l|}{ Breathing pattern and respiratory drive, median (IQR) } \\
\hline Mechanical respiratory rate, breaths/min & $25.2(18.0-29.5)$ & $25.8(21.3-32.5)$ & .99 \\
\hline Patient's own neural breathing frequency, breaths/min & $29.3(23.6-31.6)$ & $25.9(20.5-31.8)$ & .49 \\
\hline Mechanical $\mathrm{T}_{\mathrm{I}}$, $\mathrm{s}$ & $0.72(0.59-1.04)$ & $0.69(0.52-0.92)$ & .99 \\
\hline Neural $T_{I}, s$ & $0.48(0.64-0.81)$ & $0.70(0.51-0.80)$ & .62 \\
\hline Mechanical $\mathrm{T}_{\mathrm{I}} / \mathrm{T}_{\text {tot }}$ & $0.26(0.25-0.31)$ & $0.28(0.22-0.37)$ & .32 \\
\hline Neural $\mathrm{T}_{\mathrm{I}} / \mathrm{T}_{\text {tot }}$ & $0.31(0.20-0.35)$ & $0.29(0.22-0.33)$ & .99 \\
\hline Peak $\mathrm{P}_{\mathrm{aw}}, \mathrm{cm} \mathrm{H}_{2} \mathrm{O}$ & $23.0(20.7-26.3)$ & $23.3(21.2-27.1)$ & .19 \\
\hline Leaks, \% & $20.4(19.2-26.3)$ & $6.2(4.6-8.0)$ & .002 \\
\hline Peak inspiratory flow, $\mathrm{L} / \mathrm{s}$ & $1.20(1.03-1.30)$ & $2.17(1.86-2.34)$ & .002 \\
\hline Peak inspiratory flow time, $\mathrm{s}$ & $0.30(0.23-0.39)$ & $0.26(0.24-0.28)$ & .16 \\
\hline Maximum peak $\mathrm{EA}_{\mathrm{di}}, \mu \mathrm{V}$ & $8.1(4.7-12.2)$ & $5.1(3.3-10.2)$ & .19 \\
\hline \multicolumn{4}{|l|}{ Gas exchange, median (IQR) } \\
\hline $\mathrm{pH}$ & $7.43(7.41-7.45)$ & $7.43(7.42-7.45)$ & .27 \\
\hline $\mathrm{P}_{\mathrm{aO}_{2}}, \mathrm{~mm} \mathrm{Hg}$ & $86(79-88)$ & $81(78-91)$ & .62 \\
\hline $\mathrm{P}_{\mathrm{aCO}_{2}}, \mathrm{~mm} \mathrm{Hg}$ & $46(41-50)$ & $46(44-49)$ & .80 \\
\hline \multicolumn{4}{|l|}{ Pressurization and triggering performance, median (IQR) } \\
\hline $\mathrm{PTP}_{200}, \mathrm{~cm} \mathrm{H}_{2} \mathrm{O} / \mathrm{s}$ & $130(113-153)$ & $83(66-109)$ & .006 \\
\hline Index $\mathrm{PTP}_{300}, \%$ & $21.6(8.4-30.4)$ & $22.4(15.3-26.1)$ & .92 \\
\hline Index $\mathrm{PTP}_{500}, \%$ & $41.3(30.4-50.8)$ & $49.0(45.5-54.1)$ & .08 \\
\hline Inspiratory trigger delay, $\mathrm{s}$ & $0.15(0.11-0.25)$ & $0.09(0.08-0.12)$ & .037 \\
\hline Expiratory trigger delay, $\mathrm{s}$ & $0.14(0.05-0.36)$ & $0.07(0.03-0.12)$ & .16 \\
\hline Triggering phase $\mathrm{PTP}, \mathrm{cm} \mathrm{H}_{2} \mathrm{O} / \mathrm{s}$ & $5.8(2.3-9.3)$ & $1.4(1.1-2.1)$ & .006 \\
\hline Drop in airway pressure during triggering phase, $\mathrm{cm} \mathrm{H}_{2} \mathrm{O}$ & $0.80(0.60-1.05)$ & $0.52(0.37-0.85)$ & .049 \\
\hline \multicolumn{4}{|l|}{ Patient-ventilator synchrony } \\
\hline Synchronous time to neural $\mathrm{T}_{\mathrm{I}}$ ratio, median (IQR) & $0.79(0.68-0.88)$ & $0.83(0.80-0.88)$ & .37 \\
\hline Asynchrony index $>10 \%, n / N$ & $6 / 10$ & $0 / 10$ & .01 \\
\hline 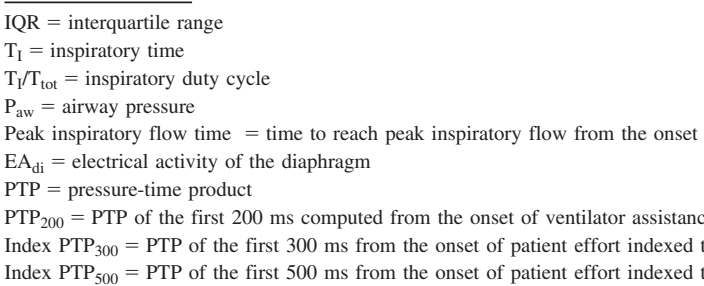 & 's effort & & \\
\hline
\end{tabular}

1-y mortality compared with the mask. ${ }^{6,21}$ In particular, the a new-generation helmet used in the present study was characterized by improved pressurization and triggering performance, ${ }^{8,9}$ which also allowed its use in more-challenging patients such as those with a COPD exacerbation. ${ }^{10}$ Use in these patients is frequently problematic due to a less-efficient reduction in inspiratory effort and poorer patient-ventilator interaction compared with a mask. ${ }^{7}$

In the present study, the time to reach peak inspiratory flow did not differ between NAVA through a helmet and pressure support through a face mask, whereas the peak inspiratory flow did. This discrepancy with a previous study, ${ }^{13}$ was probably due to the interfaces. $\mathrm{PTP}_{200}$, which is computed in the first $200 \mathrm{~ms}$ from the onset of ventilator insufflation and did not involve the triggering phase, is an index of pressurization performance of the interface; this is mainly influenced by the flow delivered from the ventilator (peak inspiratory flow) and the mechanical properties of the interface itself. During pressure support through a face mask, the interface (ie, the mask) has a very low (negligible) compliance, without any displacement during the pressurization. Therefore, the volume (which is the flow over time) delivered to the interface generates all pressurization. On the contrary, NAVA through a helmet is delivered through a helmet that is characterized by a higher compliance compared with an oronasal mask and a craniocaudal displacement during insufflation.

These also are issues with the a new-generation helmet used in the study, although less so when compared with a standard helmet. ${ }^{8,9,12,13}$ Therefore, even though during NAVA through a helmet, the flow (ie, peak inspiratory 

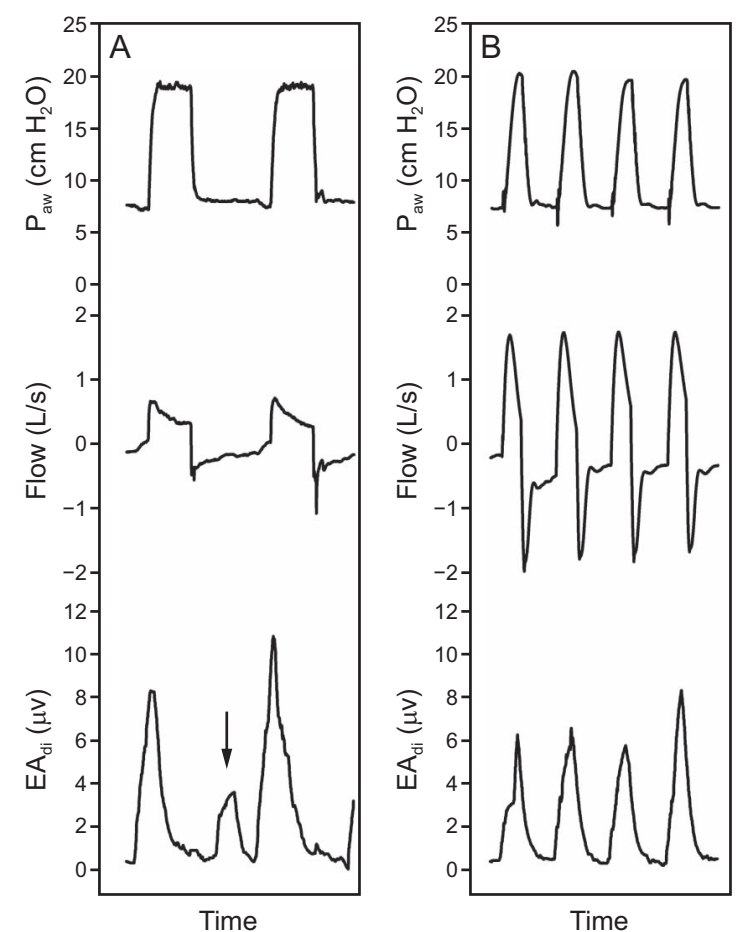

Fig. 2. Airway pressure $\left(\mathrm{P}_{\mathrm{aw}}\right)$, flow, and respiratory drive (electrical activity of the diaphragm [EAdi]) tracings are depicted of one representative subject during pneumatically triggered pressure support through a mask (A), and neurally controlled pressure support through a helmet $(B)$. The arrow indicates an ineffective inspiratory effort.

flow) is higher (and almost twice) compared with pressure support through a face mask, the flow does not generate expected pressure due to the mechanical properties of the interface (helmet). In line with this finding, it was already known that neurally triggered modes of ventilation outperform pneumatically triggered ones in subjects with COPD and with exacerbation. ${ }^{11}$ Furthermore, NAVA has also been largely demonstrated to improve patient-ventilator synchrony during both invasive ventilation ${ }^{15,18,22,23}$ and NIV. ${ }^{12,13,19,23-26}$

Our study had 2 limitations. First, we tested 2 factors altogether, that is, the interface and the triggering system, without the possibility to ascertain whether improved comfort was attributable to the new-generation helmet or the mode of ventilation, or the combination of both factors. Previous literature reports that comfort during NIV is determined by the interface, 3,5 patient-ventilator interaction and synchrony, ${ }^{23}$ and pressurization and triggering performance. ${ }^{12,13}$ However, this study seemed to indicate that comfort was improved by the interface, rather than the triggering improvement. Second, consistent with previous studies, 12,13,27,28 we applied the 11-point numeric rating scale to assess comfort, although this scale was formally only validated for pain ${ }^{29}$ and dyspnea. ${ }^{30,31}$

\section{Conclusions}

In the subjects with a COPD exacerbation and acute respiratory failure, NAVA through a helmet improved comfort, triggering performance, and patient-ventilator synchrony compared with pressure support through a face mask. It remains to be determined whether these physiologic benefits may translate into a reduced NIV failure rate and improved clinical outcomes.

\section{REFERENCES}

1. Rochwerg B, Brochard L, Elliott MW, Hess D, Hill NS, Nava S, et al. Official ERS/ATS clinical practice guidelines: noninvasive ventilation for acute respiratory failure. Eur Respir J 2017;50(2). pii: 1602426

2. Crimi C, Noto A, Princi P, Esquinas A, Nava S. A European survey of noninvasive ventilation practices. Eur Respir J 2010;36(2):362369.

3. Elliott MW. The interface: crucial for successful noninvasive ventilation. Eur Respir J 2004;23(1):7-8.

4. Vignaux L, Vargas F, Roeseler J, Tassaux D, Thille AW, Kossowsky MP, et al. Patient-ventilator asynchrony during non-invasive ventilation for acute respiratory failure: a multicenter study. Intensive Care Med 2009;35(5):840-846.

5. Antonelli M, Conti G, Pelosi P, Gregoretti C, Pennisi MA, Costa R, et al. New treatment of acute hypoxemic respiratory failure: noninvasive pressure support ventilation delivered by helmet-a pilot controlled trial. Crit Care Med 2002;30(3):602-608.

6. Patel BK, Wolfe KS, Pohlman AS, Hall JB, Kress JP. Effect of noninvasive ventilation delivered by helmet vs face mask on the rate of endotracheal intubation in patients with acute respiratory distress syndrome: a randomized clinical Trial. JAMA 2016;315(22):24352441 .

7. Navalesi P, Costa R, Ceriana P, Carlucci A, Prinianakis G, Antonelli $\mathrm{M}$, et al. Non-invasive ventilation in chronic obstructive pulmonary disease patients: helmet versus facial mask. Intensive Care Med 2007;33(1):74-81.

8. Olivieri C, Costa R, Spinazzola G, Ferrone G, Longhini F, Cammarota $\mathrm{G}$, et al. Bench comparative evaluation of a new generation and standard helmet for delivering non-invasive ventilation. Intensive Care Med 2013;39(4):734-738.

9. Olivieri C, Longhini F, Cena T, Cammarota G, Vaschetto R, Messina A, et al. New versus conventional helmet for delivering noninvasive ventilation: a physiologic, crossover randomized study in critically ill patients. Anesthesiology 2016;124(1):101-108.

10. Pisani L, Mega C, Vaschetto R, Bellone A, Scala R, Cosentini R, et al. Oronasal mask versus helmet in acute hypercapnic respiratory failure. Eur Respir J 2015;45(3):691-699.

11. Liu L, Xia F, Yang Y, Longhini F, Navalesi P, Beck J, et al. Neural versus pneumatic control of pressure support in patients with chronic obstructive pulmonary diseases at different levels of positive end expiratory pressure: a physiological study. Crit Care 2015;19:244.

12. Cammarota G, Longhini F, Perucca R, Ronco C, Colombo D, Messina A, et al. New setting of neurally adjusted ventilatory assist during noninvasive ventilation through a helmet. Anesthesiology 2016;125(6):1181-1189.

13. Longhini F, Pan C, Xie J, Cammarota G, Bruni A, Garofalo E, et al. New setting of neurally adjusted ventilatory assist for noninvasive ventilation by facial mask: a physiologic study. Crit Care 2017; 21(1): 170 .

14. Vogelmeier CF, Criner GJ, Martinez FJ, Anzueto A, Barnes PJ, Bourbeau J, et al. Global Strategy for the Diagnosis, Management, 


\section{NAVA FOR NIV IN COPD EXACERBATION}

and Prevention of Chronic Obstructive Lung Disease 2017 Report: GOLD Executive Summary. Eur Respir J 2017;49(3). pii: 1700214.

15. Vaschetto R, Cammarota G, Colombo D, Longhini F, Grossi F, Giovanniello A, et al. Effects of propofol on patient-ventilator synchrony and interaction during pressure support ventilation and neurally adjusted ventilatory assist. Crit Care Med 2014;42(1):74-82.

16. SERVO-i Ventilator System V6.0, User's Manual. Maquet Critical Care AB; 2011.

17. Cammarota G, Longhini F, Perucca R, Ronco C, Colombo D, Messina A, et al. New setting of neurally adjusted ventilatory assist during noninvasive ventilation through a helmet. Anesthesiology 2016;125(6):1181-1189.

18. Colombo D, Cammarota G, Bergamaschi V, De Lucia M, Corte FD, Navalesi P. Physiologic response to varying levels of pressure support and neurally adjusted ventilatory assist in patients with acute respiratory failure. Intensive Care Med 2008;34(11):2010-2018.

19. Cammarota G, Olivieri C, Costa R, Vaschetto R, Colombo D, Turucz $\mathrm{E}$, et al. Noninvasive ventilation through a helmet in postextubation hypoxemic patients: physiologic comparison between neurally adjusted ventilatory assist and pressure support ventilation. Intensive Care Med 2011;37(12):1943-1950.

20. Olivieri C, Costa R, Conti G, Navalesi P. Bench studies evaluating devices for non-invasive ventilation: critical analysis and future perspectives. Intensive Care Med 2012;38(1):160-167.

21. Patel BK, Wolfe KS, MacKenzie EL, Salem D, Esbrook CL, Pawlik $\mathrm{AJ}$, et al. One-year outcomes in patients with acute respiratory distress syndrome enrolled in a randomized clinical trial of helmet versus facemask noninvasive ventilation. Crit Care Med 2018;46(7): 1078-1084

22. Demoule A, Clavel M, Rolland-Debord C, Perbet S, Terzi N, Kouatchet A, et al. Neurally adjusted ventilatory assist as an alternative to pressure support ventilation in adults: a French multicentre randomized trial. Intensive Care Med 2016;42(11):1723-1732.
23. Garofalo E, Bruni A, Pelaia C, Liparota L, Lombardo N, Longhini F, Navalesi P. Recognizing, quantifying and managing patient-ventilator asynchrony in invasive and noninvasive ventilation. Expert Rev Respir Med 2018;12(7):557-567.

24. Bertrand PM, Futier E, Coisel Y, Matecki S, Jaber S, Constantin JM. Neurally adjusted ventilatory assist vs pressure support ventilation for noninvasive ventilation during acute respiratory failure: a crossover physiologic study. Chest 2013;143(1):30-36.

25. Schmidt M, Dres M, Raux M, Deslandes-Boutmy E, Kindler F, Mayaux J, et al. Neurally adjusted ventilatory assist improves patient-ventilator interaction during postextubation prophylactic noninvasive ventilation. Crit Care Med 2012;40(6):1738-1744.

26. Piquilloud L, Tassaux D, Bialais E, Lambermont B, Sottiaux T, Roeseler J, et al. Neurally adjusted ventilatory assist (NAVA) improves patient-ventilator interaction during non-invasive ventilation delivered by face mask. Intensive Care Med 2012;38(10):1624-1631.

27. Chooi CS, White AM, Tan SG, Dowling K, Cyna AM. Pain vs comfort scores after Caesarean section: a randomized trial. Br J Anaesth 2013;110(5):780-787.

28. Maggiore SM, Idone FA, Vaschetto R, Festa R, Cataldo A, Antonicelli $\mathrm{F}$, et al. Nasal high-flow versus venturi mask oxygen therapy after extubation. Effects on oxygenation, comfort, and clinical outcome. Am J Respir Crit Care Med 2014;190(3):282-288.

29. Gerbershagen HJ, Rothaug J, Kalkman CJ, Meissner W. Determination of moderate-to-severe postoperative pain on the numeric rating scale: a cut-off point analysis applying four different methods. Br J Anaesth 2011;107(4):619-626.

30. Gagliese L, Weizblit N, Ellis W, Chan VW. The measurement of postoperative pain: a comparison of intensity scales in younger and older surgical patients. Pain 2005;117(3):412-420.

31. Gift AG, Narsavage G. Validity of the numeric rating scale as a measure of dyspnea. Am J Crit Care 1998;7(3):200-204. 